\title{
Neutrino spin-flips in curved space-time
}

\author{
M. Brüggen \\ Institute of Astronomy, University of Cambridge, Madingley Road, Cambridge CB3 OHA, UK
}

\begin{abstract}
The general relativistic effects on spin-flavor oscillations above the core of type II supernovae are investigated. The evolution equation is derived and the relative magnitudes of the terms in the Hamiltonian, which arise from the weak, electromagnetic and gravitational interaction, are compared. The effects on the resonance position and the adiabaticity are studied. Explicit calculations are presented for non-rotating and slowly rotating stars.
\end{abstract}

14.60.Pq, 95.30.Sf, 26.30.+k, 97.60.Bw

Typeset using REVTEX 


\section{INTRODUCTION}

If the neutrino possesses a magnetic moment, it has been shown that its spin can be flipped while passing through a magnetic field [1] 5]. This can lead to spin-precession and spin-flavor oscillations. In the standard model of particle physics the neutrino magnetic moment is zero. However non-standard neutrino properties such as mass and magnetic moment attract enormous attention since they are not only natural by-products of many grand unified theories; but they can also lead to neutrino oscillations, which provide a very popular and elegant solution to the long-standing solar neutrino problem [6].

Terrestrial experiments constrain the magnetic moment of the neutrino to values < $10^{-10} \mu_{B}$ [7], where $\mu_{B}$ is the Bohr magneton. Astrophysical bounds are more stringent constraining $\mu<2 \times 10^{-12} \mu_{B} 1014$. The latter are, however, somewhat model-dependent.

Thus for spin-flips to be discernible, very strong magnetic fields are required. One of the places where magnetic fields of such strengths are likely to exist is above the proto-neutron star of a type II supernova (SN). Observations of magnetic fields on the surface of white dwarfs suggest the existence of magnetic fields of up to $10^{10} \mathrm{G}$ at the surface of the iron core [15]; energetic considerations suggest even higher fields [16].

In type II SNe neutrinos play a crucial role since they are believed to drive the supernova explosion [17,[18]. Neutrino oscillations could therefore affect the explosion mechanism directly. Also neutrinos from a nearby SN explosion, SN 1987 A, have been detected on Earth [19], and the measured neutrino spectrum has been used to test various oscillation scenarios 20,21. Neutrino spin-flips have also been invoked to explain the high pulsar kick velocities, as the interaction with a magnetic field can lead to an asymmetric neutrino emission [22].

It is known for some time that gyroscopes precess in strong gravitational fields [23,24]. Here I will investigate under which conditions gravitational effects can influence the neutrino spin flip in a SN.

SN explosions occur at the end of the life of massive stars and leave a neutron star or a black hole behind. Therefore it is of importance to study neutrino oscillations in 
curved space-time and calculate the magnitude of general relativistic effects. Cardall \& Fuller [25] have calculated the gravitational effect on neutrinos in vacuum and matter. They derived MSW (Mikheyev-Smirnov-Wolfenstein) oscillation formulae in curved spacetime and presented expressions for radial and azimuthal propagation in a Schwarzschild geometry. Piriz et al. [26] derived the resonance conditions for neutrino conversions with regard to neutrino oscillations in the vicinity of active galactic nuclei. They present explicit calculations for arbitrary orbits in a Kerr-geometry.

Here I will briefly rederive the evolution equation for massive Dirac neutrinos interacting with a magnetic field in matter. In Sec. 3 and 4 I will study how the neutrino conversion is affected by the curvature of space-time near a static and a slowly rotating proto-neutron star.

Throughout this paper I shall set $G=\hbar=c=1$.

\section{THE EVOLUTION EQUATION IN CURVED SPACE-TIME}

Gravitational effects on the spin arise through the "spin connection" $\Gamma_{\mu}$ appearing in the Dirac equation. This effect has been studied by Cardall \& Fuller 25] and somewhat more formally by Piriz et al. [26] (also see [27,28]). In the following I will follow the treatment of Ref. 25]. The Dirac equation in curved space-time (ignoring magnetic fields and matter effects for the moment, and restricting myself to two neutrino species) for massive neutrinos interacting minimally with the gravitational field can be written as follows 229]:

$$
\left[\gamma^{a} e_{a}^{\mu}\left(\partial_{\mu}+\Gamma_{\mu}\right)+M\right] \psi=0
$$

Here $\psi$ is a column vector of spinors of different mass eigenstates, the $\gamma^{a}$ 's represent the Dirac matrices and $M$ is the mass matrix, $M^{2}=\operatorname{diag}\left[m_{1}^{2}, m_{2}^{2}\right]$, where $m_{1}$ and $m_{2}$ are the mass eigenvalues of the two neutrino species. I follow the convention that greek indices refer to general curvilinear coordinates, while the latin indices $a, b, c, d$ refer to locally inertial (Minkowski) coordinates. The tetrads $e_{a}^{\mu}$ connect these sets of coordinates. $g_{\mu \nu}$ will 
denote the metric of curved space-time and $\eta_{a b}$ the metric in flat space, where I will use the convention that $\eta_{a b}=\operatorname{diag}[-1,1,1,1]$. The tetrads then satisfy

$$
g_{\mu \nu}=\eta_{a b} e_{\mu}^{a} e_{\nu}^{b}
$$

The explicit expression for $\Gamma_{\mu}$ is

$$
\Gamma_{\mu}=\frac{1}{8}\left[\gamma^{b}, \gamma^{c}\right] e_{b}^{\nu} e_{c \nu ; \mu}
$$

Here the semicolon denotes a covariant derivative and a comma an ordinary derivative. It can be shown that

$$
\gamma^{a}\left[\gamma^{b}, \gamma^{c}\right]=2 \eta^{a b} \gamma^{c}-2 \eta^{a c} \gamma^{b}-2 i \epsilon^{d a b c} \gamma_{5} \gamma_{d}
$$

where $\epsilon^{a b c d}$ is the (flat space) totally antisymmetric tensor, with $\epsilon^{0123}=+1$. With Eq. (丑), the non-vanishing contribution from the spin connection is

$$
\gamma^{a} e_{a}^{\mu} \Gamma_{\mu}=\gamma^{a} e_{a}^{\mu}\left\{i A_{G \mu}\left[-\frac{\gamma_{5}}{2}\right]\right\}
$$

where

$$
A_{G}^{\mu} \equiv \frac{1}{4} e_{a}^{\mu} \epsilon^{a b c d}\left(e_{b \nu, \sigma}-e_{b \sigma, \nu}\right) e_{c}^{\nu} e_{d}^{\sigma}
$$

The expression in Eq. (5) treats left- and right-handed states differently. In order to group it with terms arising from matter effects, we can without physical consequence add a term proportional to the identity to obtain

$$
\gamma^{a} e_{a}^{\mu} \Gamma_{\mu}=\gamma^{a} e_{a}^{\mu}\left(i A_{G \mu} \mathcal{P}_{L}\right)
$$

where $\mathcal{P}_{L}$ is the left-handed projection operator $\mathcal{P}_{L}=\left(1-\gamma^{5}\right) / 2$. As pointed out by Cardall \& Fuller [25], $A_{G \mu}$ is diagonal in spin space and cannot produce spin flips on its own. However it can change the resonance as well as the adiabaticity conditions in the presence of other off-diagonal terms such as those arising from an interaction with a magnetic field.

A further complication arises in curved space-time: The neutrino trajectories are no longer necessarily straight lines as in flat space-time. They can be written as curves of the 
affine parameter $\lambda$. The tangent vectors to the null worldline, $p^{\mu}=d x^{\mu} / d \lambda$, can be found by solving the geodesic or Hamilton-Jacobi equation (see, e.g., [30]). As suggested in Ref. 25] the four-momentum $P_{\mu}$ is then constructed by assuming that the neutrinos are energy eigenstates and that the three momentum is collinear with $\left(p_{1}, p_{2}, p_{3}\right)$.

The mass matrix $M$ is related to the mass matrix in flavor space $M_{f}$ through a rotation by the vacuum mixing angle $\theta_{v}$ as follows:

$$
M_{f}^{2}=U\left(\begin{array}{cc}
m_{1}^{2} & 0 \\
0 & m_{2}^{2}
\end{array}\right) U^{\dagger}
$$

with

$$
U=\left(\begin{array}{cc}
\cos \theta_{v} & \sin \theta_{v} \\
-\sin \theta_{v} & \cos \theta_{v}
\end{array}\right) .
$$

The neutrinos interact with matter through a weak interaction current of the form: $A_{w}^{\mu} \mathcal{P}_{L}$, where in the chiral basis $\left(\nu_{e L}, \nu_{e R}, \nu_{\mu L}, \nu_{\mu R}\right): A_{w}^{\mu}=\operatorname{diag}\left[V_{c}+V_{n}, V_{n}, 0,0\right]$ (Here the $\nu_{\mu} \mathrm{s}$ can, of course, be replaced by $\nu_{\tau} \mathrm{s}$.). The matter potentials due to the neutral and charged current in neutral, unpolarized matter are given by $V_{n}=-\sqrt{2} G_{F} n_{n}$ and $V_{c}=2 \sqrt{2} G_{F} n_{e}$, respectively, where $G_{F}$ is Fermi's constant and $E_{l}$ the energy as measured in a local inertial frame. $n_{e}$ and $n_{n}$ are the electron and neutron density in the rest frame of the background matter.

The interaction of the neutrino with an external magnetic field is described by a term of the form

$$
\hat{\mu} \sigma^{a b} F_{a b} \psi
$$

where $\hat{\mu}$ is the magnetic moment matrix, $F_{a b}$ the electromagnetic field tensor and $\sigma^{a b}=$ $\frac{1}{4}\left[\gamma^{a}, \gamma^{b}\right]$

Only considering conversions between two neutrino flavors we can write the evolution equation as follows: 


$$
i \frac{d}{d \lambda}\left(\begin{array}{c}
\nu_{e L} \\
\nu_{\mu L} \\
\nu_{e R} \\
\nu_{\mu R}
\end{array}\right)=\hat{H}\left(\begin{array}{c}
\nu_{e L} \\
\nu_{\mu L} \\
\nu_{e R} \\
\nu_{\mu R}
\end{array}\right)
$$

where $\hat{H}$ is the Hamiltonian in the chiral basis. Including the weak, electromagnetic and gravitational interaction the Hamiltonian can be written as:

$$
\hat{H}=\left(\begin{array}{cccc}
V_{c}+V_{n}+P_{\mu} A_{G}^{\mu} / E_{l}-\frac{\delta m^{2}}{4 E_{l}} \cos 2 \theta_{v} & \frac{\delta m^{2}}{4 E_{l}} \sin 2 \theta_{v} & \mu_{e e} B & \mu_{e \mu} B \\
\frac{\delta m^{2}}{4 E_{l}} \sin 2 \theta_{v} & V_{n}+P_{\mu} A_{G}^{\mu} / E_{l}+\frac{\delta m^{2}}{4 E_{l}} \cos 2 \theta_{v} & \mu_{\mu e} B & \mu_{\mu \mu} B \\
\mu_{e e} B & \mu_{e \mu} B & -\frac{\delta m^{2}}{4 E_{l}} \cos 2 \theta_{v} & \frac{\delta m^{2}}{4 E_{l}} \sin 2 \theta_{v} \\
\mu_{\mu e} B & \mu_{\mu \mu} B & \frac{\delta m^{2}}{4 E_{l}} \sin 2 \theta_{v} & \frac{\delta m^{2}}{4 E_{l}} \cos 2 \theta_{v}
\end{array}\right)
$$

where $B$ is the component of the magnetic field which is perpendicular to the neutrino trajectory and $\delta m^{2}$ the difference of the squares of the neutrino mass eigenvalues in matter: $\delta m^{2}=m_{2}^{2}-m_{1}^{2}$. Here it was assumed that the right-handed components are sterile and do not interact with matter. Resonance occurs whenever the diagonal elements cross. From the expression for $\hat{H}$ we can immediately read off the resonance conditions for spin-flips and MSW-conversions:

For $\nu_{e L} \leftrightarrow \nu_{\mu L}$ :

$$
V_{c}-\frac{\delta m^{2}}{2 E_{l}} \cos 2 \theta_{v}=0,
$$

for $\nu_{e L} \leftrightarrow \nu_{e R}$ :

$$
V_{c}+V_{n}+P_{\mu} A_{G}^{\mu} / E_{l}=0,
$$

for $\nu_{e L} \leftrightarrow \nu_{\mu R}$ :

$$
V_{c}+V_{n}+P_{\mu} A_{G}^{\mu} / E_{l}-\frac{\delta m^{2}}{2 E_{l}} \cos 2 \theta_{v}=0,
$$

for $\nu_{\mu L} \leftrightarrow \nu_{e R}$ : 


$$
V_{n}+P_{\mu} A_{G}^{\mu} / E_{l}+\frac{\delta m^{2}}{2 E_{l}} \cos 2 \theta_{v}=0
$$

and for $\nu_{\mu L} \leftrightarrow \nu_{\mu R}$ :

$$
V_{n}+P_{\mu} A_{G}^{\mu} / E_{l}=0
$$

These equations are similar to those derived in Ref. [26]. In the active-active MSW transition, Eq. (13), the matter potential is due to the charged current only and proportional to $Y_{e}$. [ $Y_{e}$ is the electron fraction: $Y_{e}=n_{e} /\left(n_{n}+n_{e}\right)$.] However for the transitions corresponding to Eqs. (14) and (15) the potential is due to the neutral as well as the charged current and thus proportional to $\left(1-2 Y_{e}\right)$. The latter potential is strongly suppressed in the isotopically neutral region (number of protons=number of neutrons) 33.].

In the following sections I will calculate the 'gravitational current', $P_{\mu} A_{G}^{\mu}$, for a static as well as for a rotating star.

\section{RELATIVISTIC EFFECTS NEAR STATIC STAR}

The geometry in a spherically symmetric, static spacetime can be globally represented by the Schwarzschild coordinate system $\left\{x^{\mu}\right\} \Rightarrow(t, r, \theta, \phi)$. MSW transitions in a Schwarzschild metric have already been discussed in Ref. 25] and their findings can easily be extended to spin-flip conversions. The Schwarzschild line element, which serves to define these coordinates, is

$$
\begin{aligned}
d s^{2}= & g_{\mu \nu} d x^{\mu} d x^{\nu} \\
= & -e^{2 \Phi(r)} d t^{2}+e^{2 \Lambda(r)} d r^{2} \\
& +r^{2} d \theta^{2}+r^{2} \sin ^{2} \theta d \phi^{2} .
\end{aligned}
$$

Using the tetrads

$$
e_{a}^{\mu}=\operatorname{diag}\left[e^{-\Phi(r)}, e^{-\Lambda(r)}, \frac{1}{r}, \frac{1}{r \sin \theta}\right]
$$


it is easy to verify that $A_{G}^{\mu}=0$, as pointed out by [25]. In Schwarzschild metric it is common to write $e^{2 \Phi(r)}=1-r_{s} / r$ and $e^{2 \Lambda(r)}=1 /\left(1-r_{s} / r\right)$, where the Schwarzschild radius $r_{s}=2 \mathcal{M}$ and $\mathcal{M}$ is the central gravitating mass. The energy-like component of the four-momentum is the energy and a conserved quantity: $P_{0}=-E$. The energy measured by a locally inertial observer is $E_{l}=E e^{-\Phi(r)}$; i.e., the energy of the neutrino suffers a gravitational redshift by $1 / \sqrt{1-r_{s} / r}$ [34. Thus the resonance conditions are merely altered by setting $E_{l}$ equal to $E e^{-\Phi(r)}$, and $A_{G}^{\mu}=0$.

For a particular transition one can define a helicity mixing angle, $\theta_{m}$, which diagonalises the corresponding submatrix in the Hamiltonian (see, e.g., [6]). For instance, for the transition $\nu_{e L} \leftrightarrow \nu_{\mu R}$, the mixing angle is defined by:

$$
\tan 2 \theta_{m}=\frac{2 \mu_{e \mu} B}{e^{\Phi} \delta m^{2} \cos 2 \theta_{v} / 2 E-\left(V_{c}+V_{n}\right)} .
$$

The adiabaticity parameter corresponding to a particular transition, $\gamma$, quantifies the magnitude of the off-diagonal elements compared to the diagonal ones of $\hat{H}$ in the basis of the instantaneous eigenstates. At the resonance point it must be greater than 1 for adiabatic propagation. $\gamma$ is modified by relativistic effects, and for the case $\nu_{e L} \leftrightarrow \nu_{\mu R}$, can be written as:

$$
\gamma\left(r_{r e s}\right)=\frac{8 e^{-\Phi\left(r_{r e s}\right)} E \mu_{e \mu}^{2} B^{2}}{\delta m^{2} \cos 2 \theta_{v} e^{-\Lambda\left(r_{r e s}\right)}\left|d \ln \left(V_{c}+V_{n}\right) / d r\right|}
$$

where the logarithmic derivative is evaluated at the resonance position. Thus the adiabaticity of the evolving neutrino is affected by the spatial dependence of the metric, which appears for example in the determination of the local energy. The conversion probability can then be expressed as 6

$$
P_{\nu_{e L} \rightarrow \nu_{\mu R}}=\frac{1}{2}-\left(\frac{1}{2}-P_{h o p}\right) \cos 2 \theta_{v} \cos 2 \theta_{m}
$$

where the non-adiabatic hopping probability is given by $P_{h o p}=\exp \left[-\frac{\pi}{2} \gamma\left(r_{\text {res }}\right)\right]$.

The conversion probabilities corresponding to the other transitions of Eqs. (13-17) can be found accordingly. 


\section{RELATIVISTIC EFFECTS NEAR ROTATING STAR}

Rotation of the collapsing star will cause a dragging of the inertial frames and will result in off-diagonal terms in the metric $g_{\mu \nu}$. These off-diagonal terms can now flip the spin of the neutrinos by transferring the angular momentum of the star to the neutrino spin. Using perturbation theory one can write down a metric to first order in the angular velocity of rotation $\Omega$ of the central mass (e.g., see [31, 32]):

$$
\begin{aligned}
d s^{2}= & -e^{2 \Phi(r)} d t^{2}+e^{2 \Lambda(r)} d r^{2} \\
& +r^{2}\left(d \theta^{2}+\sin ^{2} \theta d \phi^{2}\right)-2 \omega r^{2} \sin ^{2} \theta d \phi d t
\end{aligned}
$$

where $\omega$ is the local precession frequency and can be written as $\omega=2 J / r^{3}$ [31], where $J$ is identified with the angular momentum of the central gravitating mass. Here it is assumed that the angular momentum vector of the star, $\mathbf{J}$, is aligned with the polar axis. The metric of Eq. (23) is valid as long as the rotation is slow, i.e., for $\Omega a<<1$, where $a$ is the radius of the central mass.

In this metric purely radial motion is only possible along the axis of rotation as expected from symmetry considerations. From these same considerations it is also evident that motion in a plane is only possible in the equatorial plane.

However, here I will confine myself to nearly radial trajectories and work in the weakfield limit so that the curvature of the neutrino path can be neglected. Thus one finds from Eqs. (6) and (23):

$$
P_{\mu} A_{G}^{\mu} / E_{l} \approx e^{-\Lambda(r)} J r^{-3} \cos \theta
$$

for relativistic neutrinos moving radially outwards (i.e. $P_{1} \approx P_{0}$ ). It can be noted that for propagation in the equatorial plane the 'gravitational current' is still zero. But at any other polar angle there will now be an additional term in the Hamiltonian which will shift the resonance position and modify the adiabaticity conditions. In Eqs. (20) and (21) one can then simply replace $V_{c}+V_{n}$ by $V_{c}+V_{n}+P_{\mu} A_{G}^{\mu} / E_{l}$. It is interesting to note that $P_{\mu} A_{G}^{\mu}$ 
changes sign at the equator and could thus lead to an asymmetry in the neutrino emission. It is evident from Eq. (24) that the 'gravitational current' moves the resonance point closer to the center of the SN in the upper hemisphere and farther away in the lower hemisphere. The magnitude of this effect will be discussed in the following section.

\section{DISCUSSION}

Simple angular momentum arguments suggest high angular velocities for neutron stars, which have also been verified by observations. During collapse to a neutron star the angular velocity of a typical star will increase from about $\Omega_{0} \approx 10^{-6} \mathrm{~s}^{-1}$ to $\Omega \approx 10^{4} \mathrm{~s}^{-1}$. Fig. 1 shows the absolute magnitudes of the various components in the Hamiltonian given by Eq. (12) for a neutrino propagating in a typical SN environment. I consider here the case when the protoneutron star is rotating, as $A_{G}=0$ otherwise. The neutrino is assumed to move radially along the polar axis. The matter potential was calculated using the progenitor model for a $15 M_{\odot}$, low metallicity star as calculated in Ref. [16] on the basis of the models of Ref. [35]. It can be seen that in the region between $0.02 R_{\odot}$ and $0.3 R_{\odot}$ the matter potential is strongly suppressed due to the high abundance of isotopic elements such as Oxygen, Carbon and Helium.

The magnetic field was assumed to be a dipole field which is aligned with the rotation axis and is given by:

$$
B=B_{0}\left(\frac{r_{0}}{r}\right)^{3} \sin \theta,
$$

where $B_{0}$ is the magnitude of the magnetic field at the surface of the iron core at a radius

$r_{0}$. The value of the neutrino magnetic moment was taken to be $\mu=10^{-12} \mu_{B}$ and $B_{0}$ was set to $10^{10} \mathrm{G}$. The 'gravitational current' was calculated assuming that the iron core has collapsed to form a proto-neutron star of mass $M=2 M_{\odot}$ and a radius of $a=10 \mathrm{~km}$. Its angular velocity was taken to be $\Omega=10^{6} \mathrm{~s}^{-1}$. It is seen that the 'gravitational current' comes relatively close to the matter potential in the isotopically neutral region of the $\mathrm{SN}$ 
envelope. Also shown in Fig. 1 is the absolute difference of the diagonal elements of the Hamiltonian for the transition $\nu_{e L} \leftrightarrow \nu_{\mu R}$, i.e. the right-hand side of Eq. (14). Resonance occurs whenever the difference in the diagonal elements goes through zero. It is evident that in order for the 'gravitational current' to affect the neutrino spin-flip, the resonant transition has to take place in the isotopically neutral region. For a choice of $\delta m^{2}=10^{-10} \mathrm{eV}^{2}$ and $\cos \theta_{v} \approx 1$ (large mixing angle) the resonance occurs at about $r=0.15 R_{\odot}$.

For magnetic fields as high as indicated in Fig. 1 the off-diagonal term, $\mu B$, dominates the potential by several orders of magnitude such that in the isotopically neutral region spin-flavor precession of almost maximum depth occurs. Strong precession occurs whenever $\mu B$ is much greater than $\Delta H$ and the precession amplitude is given by [16]:

$$
A_{p}=\frac{(2 \mu B)^{2}}{(2 \mu B)^{2}+\Delta H^{2}}
$$

In that case the gravitational current has a negligible effect on the conversion probability. However for weaker - and possibly more realistic - magnetic fields $\left(B_{0}<10^{7} \mathrm{G}\right) \mu B$ becomes comparable to $\Delta H$, suppressing the spin-flavor precession. However $\mu B$ is still greater than $\Delta H$ at the resonance layer, where resonant conversion occurs as long as the adiabaticity condition is satisfied.

It is readily seen that for our example the 'gravitational current' is still by about one order of magnitude smaller than the matter potential and will thus not significantly affect the resonance position or the adiabaticity in the envelope of the $\mathrm{SN}$ (even for $B_{0}<10^{7} \mathrm{G}$ ) .

However if the mass of the proto-neutron star or its rotation rate is increased, or the matter potential in the envelope is suppressed by about one order of magnitude (i.e., by reducing the abundance of high $\mathrm{Z}$ metals), the presence of the gravitational current will start to shift the resonance position and affect the transition probability noticably.

The conditions for which the gravitational contribution becomes important are neither contrived nor unrealistic. These calculations are only intended to yield crude orders of magnitude estimates for the different terms in the evolution equation and more exact numbers would require a more careful modelling of the collapsing star. For instance, I have neglected 
the infall velocity of the matter during the collapse and only considered radial neutrino trajectories in the weak-field limit. However these simple estimates presented here show that the terms arising from the space-time curvature can potentially become significant for spin-flip transitions in the SN envelope even though they are probably not important in most realistic cases. The extent to which they affect the neutrino transition depends on the

detailed composition of the $\mathrm{SN}$, as well as the mass and rotation rate of the proto-neutron star.

\section{ACKNOWLEDGMENTS}

I am grateful to Dr. T. Totani for providing me with the data for the progenitor models. 


\section{REFERENCES}

[1] C.S. Lim and W.J. Marciano, Phys. Rev. D 37, 1368 (1988).

[2] M.B. Voloshin, Phys. Lett. B 209, 360 (1988).

[3] E.Kh. Akhmedov, Phys. Lett. B 213, 64 (1988).

[4] J. Schechter and J.W.F. Valle, Phys. Rev. D 24, 1883 (1981); erratum: Phys. Rev. D 25, $283(1982)$.

[5] M.B. Voloshin, M.I. Vysotsky and L.B. Okun, Zh. Eksp. Teor. Fiz. 91, 754 (1986) [Sov. Phys. JETP 64, 446 (1986)].

[6] W.C. Haxton, Ann. Rev. Astron. Astrophys. 33, 459 (1995).

[7] Particle Data Group, R.M. Barnett et al., Phys. Rev. D 54, 1658 (1996).

[8] G.S. Vidyakin et al., JETP Lett. 55, 206 (1992).

[9] P. Vogel and J. Engel, Phys. Rev. D 39, 3378 (1989).

[10] D. Nötzold, Phys. Rev. D 38, 1658 (1988).

[11] J.T. Peltoniemi, Astron. Astrophys. 254, 121 (1992).

[12] J.M. Lattimer and J. Cooperstein, Phys. Rev. Lett. 61, 23 (1988).

[13] G.G. Raffelt and A. Weiss, Astron. Astrophys. 264, 536 (1992).

[14] G.G. Raffelt, Phys. Rev. Lett. 64, 2856 (1990).

[15] G. Chanmugam, Annu. Rev. Astron. Astrophys. 30, 143 (1992).

[16] T. Totani and K. Sato, Phys. Rev. D 54, 5975 (1996).

[17] H.A. Bethe and J.R. Wilson, Astrophys. J. 295, 14 (1985).

[18] A.S. Burrows, in Supernovae, edited by A.G. Petschek, (Springer-Verlag, New York,1990). 
[19] K.S. Hirata et al., Phys. Rev. Lett. 58, 1490 (1987); R.M. Bionta et al., Phys. Rev. Lett. 58, 1494 (1987).

[20] T.J. Loredo and D.Q. Lamb, in Proceedings of the 14th Texas Symposium on Relativistic Astrophysics, Dallas, Texas, 1988, edited by E. Fenyves [Ann. (N.Y.) Acad. Sci. 571, $(1989)]$.

[21] A.Yu. Smirnov, D.N. Spergel, and J.N. Bahcall, Phys. Rev. D 49, 1389 (1994).

[22] E.Kh. Akhmedov, A. Lanza, D.W. Sciama, hep-ph/9702436 (1997)

[23] H. Thirring, Phys. Zs. 19, 33 (1918).

[24] D. Brill and J. Cohen, Phys. Rev. 143, 1011 (1966).

[25] C.Y. Cardall and G.M. Fuller, Phys. Rev. D 55, 7960 (1996).

[26] D. Píriz, M. Roy, and J. Wudka, Phys. Rev. D 54, 1587 (1996).

[27] D. V. Ahluwalia and C. Burgard, LA-UR-96-862, gr-qc/9603008 (1996); D.V. Ahluwalia and C. Burgard, LA-UR-96-2031, gr-qc/9606031 (1996).

[28] Y. Grossman and H.J. Lipkin, WIS-96/27/Jun-PH, TAUP 2346-96, hep-ph/9607201 (1996).

[29] See e.g. S.W. Weinberg, Gravitation and Cosmology (Wiley, New York, 1972), Sec. 12.5; D.R. Brill and J.A. Wheeler, Rev. Mod. Phys. 29, 465.

[30] L.D. Landau and E.M. Lifshitz, Classical Theory of Fields, Oxford (1975)

[31] J.B. Hartle, Astrophys. J. 150, 1005 (1967).

[32] C.W. Misner, K.S. Thorne, and J.A. Wheeler Gravitation, p. 699 Freeman, New York (1973).

[33] H. Athar, J.T. Peltoniemi, and A.Yu. Smirnov, Phys. Rev. D, 51, 6647 (1995). 
[34] G.M. Fuller and Y.-Z. Qian, Nucl. Phys. A 606, 167 (1996).

[35] S.E. Woosley and T.A. Weaver, Astrophys. J. Suppl. Ser. 101, 181 (1995). 


\section{FIGURES}

FIG. 1. Magnitudes of the various components in the Hamiltonian of (12) as a function of distance from the center of a $15 M_{\odot}$ type II SN. The energies are measured in $\mathrm{eV}$ and the radius is measured in units of solar radii. The lines represent the matter potential (solid), the gravitational term (short-dash), the magnetic term (dotted) and the absolute difference of the diagonal elements in the Hamiltonian (long-dash). 


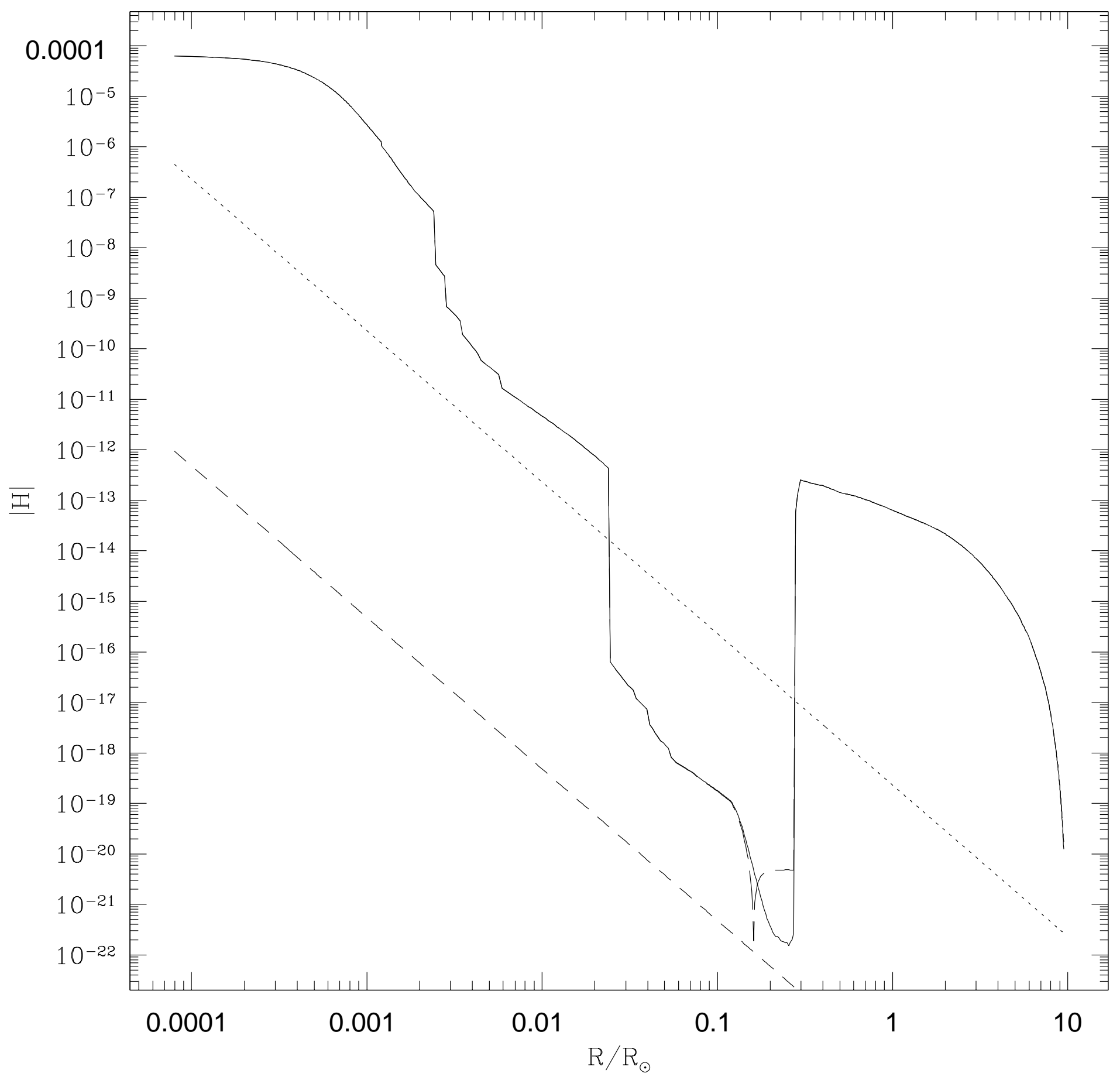

